\title{
Skewon no-go theorem
}

\author{
Yakov Itin \\ Institute of Mathematics, The Hebrew University of Jerusalem \\ and Jerusalem College of Technology, Jerusalem, Israel. \\ email: itin@math.huji.ac.il
}

(Dated: May 27, 2022)

\begin{abstract}
Axion modification of the electrodynamics can be considered as produced by an irreducible part of the constitutive pseudotensor. In this paper, we study the modification of wave propagation produced by the second irreducible part called skewon. We introduce the notions of skewon optic tensor and skewon optic covector. With these devices we prove that in a pseudo-Riemannian manifold endowed with an arbitrary skewon at least one solution of the dispersion relation is spacelike. This means that the skewon generates superluminal wave motion and is thus ruled out on the basis of SR principles.
\end{abstract}

PACS numbers: 75.50.Ee, 03.50.De, 46.05.+b, 14.80.Mz

Although Maxwell electromagnetism is a theoretically well established and experimentally confirmed physical theory, its modifications are frequently considered. In particular, axion modified electrodynamics was proposed in [1] and [2]. A much richer class of CPT-violation models of electrodynamics was studied in [3] and [4]. In [5] we show that axion can be viewed as an irreducible part of a generic constitutive pseudo-tensor. Although it does not enter the wave propagation in the geometrical optics description it contributes to the higher approximation. As it is shown in [6], axion yields very hard deformations of the light cone structure and thus can be ruled out on the basis of SR first principles. In this paper, we consider the modifications of the light cone structure by the skewon, that is the second irreducible part of the constitutive pseudo-tensor [7], [8], [9], [10], [11].

In non-dissipative linear response media, the electromagnetic field can be described by a covariant Maxwell system $[7]$

$$
\epsilon^{i j k l} F_{i j, k}=0 \quad H^{i j}{ }_{, j}=J^{i}
$$

with a linear homogeneous constitutive relation between the excitation $H^{i j}$ and the field strength $F_{i j}$,

$$
H^{i j}=\frac{1}{2} \chi^{i j k l} F_{k l} .
$$

Due to its definition, the constitutive pseudo-tensor $\chi^{i j k l}$ inherits the symmetries of the skew-symmetric tensors $H^{i j}$ and $F_{i j}$,

$$
\chi^{i j k l}=-\chi^{j i k l}=-\chi^{i j l k} .
$$

In four dimensional space, $\chi^{i j k l}$ has therefore 36 independent components. Under the action of the group $G L(4, \mathbb{R})$, such a fourth order tensor possesses a unique irreducible decomposition into the sum of three independent pieces:

$$
\chi^{i j k l}={ }^{(1)} \chi^{i j k l}+{ }^{(2)} \chi^{i j k l}+{ }^{(3)} \chi^{i j k l} .
$$

Here, the third tensor is totally skew-symmetric, so it has only one independent component. Writing it as

$$
{ }^{(3)} \chi^{i j k l}=\chi^{[i j k l]}=\alpha \varepsilon^{i j k l}
$$

the axion pseudo-scalar $\alpha$ emerges. This hypothetic copartner of the photon was intensively discussed in the literature.

The second tensor of (0.4) is given by

$$
{ }^{(2)} \chi^{i j k l}=\frac{1}{2}\left(\chi^{i j k l}-\chi^{k l i j}\right)
$$

This skewon tensor of 15 components was introduced only recently in [7].

The first tensor, called the principal part of $\chi^{i j k l}$, has 20 independent components. It is expressed as

$$
\begin{aligned}
{ }^{(1)} \chi^{i j k l}= & \frac{1}{6}\left(2\left(\chi^{i j k l}+\chi^{k l i j}\right)-\left(\chi^{i k l j}+\chi^{l j i k}\right)\right. \\
& \left.-\left(\chi^{i l j k}+\chi^{j k i l}\right)\right) .
\end{aligned}
$$

In ordinary Maxwell electrodynamics and its extension to curved manifolds of GR, the principal part is constructed from the metric tensor

$$
{ }^{(1)} \chi^{i j k l}=\sqrt{-g}\left(g^{i k} g^{j l}-g^{i l} g^{j k}\right) .
$$

In this case, the two other pieces ${ }^{(2)} \chi^{i j k l}$ and ${ }^{(3)} \chi^{i j k l}$ are equal to zero. In a non-relativistic anisotropic linear response material, ${ }^{(1)} \chi^{i j k l}$ decomposes into the standard sets of 3-dimensional electric and magnetic parameters.

Thus the principle part can be considered as a set of classical parameters aggregated into a covariant 4dimensional tensor. In contrast, the axion and skewon tensors do not have classical analogs. Their existence (or non-existence) is an intriguing problem for both theoretical analysis and experimental investigations. Moreover, if such additional electromagnetic parameters could be implemented in artificial materials, they might have important applications for modern technology.

In order to study light propagation in a generic medium equipped with the whole set of constitutive constants, one applies the geometric optic approximation [7], [12], [13]. Here the system of differential equations (0.1) is substituted by the the system of 8 algebraic equations

$$
\varepsilon^{i j k l} q_{j} f_{k l}=0, \quad \chi^{i j k l} q_{j} f_{k l}=0,
$$


where $q_{i}=\partial \varphi / \partial x^{i}$ is the wave covector corresponding to the wavefront $\varphi\left(x^{i}\right)=0$. The components of the tensor $f_{k l}$ are constructed from the limiting values of the true field strength $F_{k l}$. When the Fourier transforms exist, they turn into the phasors of $F_{k l}$.

The first equation of (0.9) is easily solved:

$$
f_{k l}=\frac{1}{2}\left(a_{k} q_{l}-a_{l} q_{k}\right)
$$

where $a_{k}$ is an arbitrary covector, which is an algebraic analog of the electromagnetic potential. Substituting (0.10) into the second equation of (0.9) we obtain the characteristic system

$$
M^{i k} a_{k}=0
$$

where the characteristic matrix is given by

$$
M^{i k}=\chi^{i j k l} q_{j} q_{l}
$$

Observe the straightforward identities

$$
M^{i k} q_{k}=0 \quad \text { and } \quad M^{i k} q_{i}=0
$$

which are consequences of the gauge invariance of our system. Thus the matrix $M^{i k}$ is singular. The system (0.11) has a trivial solution proportional to $q_{k}$ which does not contribute to field strength. In order to have a physically meaningful solution, the condition

$$
\operatorname{adj}(M)=0
$$

must be applied [15]. Recall the standard expression of the fourth order adjoint matrix $\left(\operatorname{adj}(M)=A_{i j}\right)$

$$
A_{i j}=\frac{1}{3 !} \varepsilon_{i i_{1} i_{2} i_{3}} \varepsilon_{j j_{1} j_{2} j_{3}} M^{i_{1} j_{1}} M^{i_{2} j_{2}} M^{i_{3} j_{3}}
$$

Consequently, the dispersion relation (0.14) reads $A_{i j}=$ 0 or

$$
\varepsilon_{i i_{1} i_{2} i_{3}} \varepsilon_{j j_{1} j_{2} j_{3}} M^{i_{1} j_{1}} M^{i_{2} j_{2}} M^{i_{3} j_{3}}=0 .
$$

It turns out [15], that we have only one independent relation here. Indeed, for a singular matrix satisfying (0.13), the adjoint matrix $A_{i j}$ is proportional to the tensor product of $q_{i}$,

$$
A_{i j}=\lambda(q) q_{i} q_{j}
$$

Consequently, the covariant dispersion relation is represented by a scalar equation

$$
\lambda(q)=0
$$

where $\lambda(q)$ is a 4 -th order polynomial in $q$ and a 3 -rd order polynomial in $\chi$.

Wave propagation is completely defined by the tensor $M^{i k}$, which is an analog of the acoustic (Christoffel) $3 \times 3$ matrix and can be referred to as optic tensor. Substituting the decomposition (0.4) into (0.13), we have

$$
M^{i k}={ }^{(1)} \chi^{i j k l} q_{j} q_{l}+{ }^{(2)} \chi^{i j k l} q_{j} q_{l}+{ }^{(3)} \chi^{i j k l} q_{j} q_{l} .
$$

Observe that because of the skew-symmetry of ${ }^{(3)} \chi^{i j k l}$ the last term is identically zero, so the axion part does not contribute to wave propagation. Indeed the known role of the axion as a possible source of CPT-violation [2] appears only beyond the geometrical optic approximation [5]. The two remaining terms on the right hand side of (0.19) are in general non zero. Consequently the optic matrix decomposes as

$$
M^{i k}=P^{i k}+Q^{i k}
$$

Here, the principle optic tensor $P_{i k}$ and the skewon optic tensor $Q_{i k}$ are defined by

$$
P^{i k}={ }^{(1)} \chi^{i j k l} q_{j} q_{l}, \quad Q^{i k}={ }^{(2)} \chi^{i j k l} q_{j} q_{l} .
$$

It follows from these definitions that these tensors satisfy

$$
P^{i k}=P^{k i}, \quad Q^{i k}=-Q^{k i} .
$$

Moreover, they are both singular

$$
P^{i k} q_{k}=0, \quad \text { and } \quad Q^{i k} q_{k}=0 .
$$

Since the second equation of (0.23) involves a skewsymmetric matrix, it can be easily solved

$$
Q^{i k}=\varepsilon^{i j k l} q_{j} Y_{l}
$$

Here the covector $Y_{i}$ is a 1-st order polynomial in $q$. It is only defined up to the addition of an arbitrary wave covector $q_{i}$. Thus an additional gauge fixing condition can be imposed on $Y_{i}$.

Since skewon part of the constitutive tensor has 15 independent components, it can be expressed as a traceless quadratic matrix. Such representation was derived in [7]:

$$
{ }^{(2)} \chi^{i j k l}=\varepsilon^{i j m k} S_{m}{ }^{l}-\varepsilon^{i j m l} S_{m}{ }^{k} .
$$

Here $S_{m}{ }^{k}$ is a traceless tensor, $S_{m}{ }^{m}=0$. Correspondingly, the skewon optic tensor is represented as

$$
Q^{i k}=\varepsilon^{i j m k} S_{m}^{l} q_{j} q_{l}
$$

Rearranging the indices we write it as

$$
Q^{i j}=\varepsilon^{i j k l} S_{l}^{m} q_{k} q_{l} .
$$

and compare with the definition of the skewon covector (0.24). Consequently we have

$$
Y_{i}=S_{i}{ }^{j} q_{j}+\alpha q_{i}
$$

where $\alpha$ is an arbitrary zero order homogeneous function of the wave covector $q$. Different choices of the parameter $\alpha$ represent different gauge conditions.

Substituting (0.20) and (0.24) into (0.18) and calculating the adjoint we obtain that the dispersion relation for a most generic linear constitutive pseudo-tensor reads

$$
\lambda(P)+P^{i j} Y_{i} Y_{j}=0 .
$$


We will study this equation for a medium with a simple principal part generated by a metric (0.8) and with a generic skewon part ${ }^{(2)} \chi^{i j k l}$. Substituting (0.8) into (0.29) we derive the following very simple form of the dispersion relation

$$
q^{4}=Y^{2} q^{2}-(Y q)^{2}
$$

Note that this equation is preserved when a "gauge transformation" $Y_{i} \rightarrow Y_{i}+\alpha q_{i}$ with an arbitrary $\alpha$ is applied. So we can write it in an even simpler form by applying a gauge condition on $Y_{i}$. We chose the Lorenz-type gauge condition

$$
(Y q)=0
$$

and we are left with the dispersion relation

$$
q^{4}=Y^{2} q^{2}
$$

This equation is not as trivial as it first appears. Note that the covector $Y_{i}$ is a 1-st order homogeneous function of $q_{i}$, but it is not necessarily a polynomial. Indeed, in the Lorenz-type gauge $(0.32)$, the optic covector $(0.28)$ is expressed as

$$
Y_{i}=S_{i}{ }^{j} q_{j}-\frac{S^{m n} q_{m} q_{n}}{q^{2}} q_{i} .
$$

In general, the second term here is equal to zero. See, for instance, the spatially isotropic skewon field of Nieves and Pal [8] with $S_{i}{ }^{j} \sim \operatorname{diag}(-3,1,1,1)$. Another explicit examples of such behaviors are exhibited in [9]. Thus (0.32) does not necessary have a light-like solution $q^{2}=0$.

In order to analyze the solutions of $(0.32)$, we first observe that its left hand side is nonegative. Thus in the case $q^{2} \neq 0$, the "squares" $Y^{2}$ and $q^{2}$ have the same sign. The covectors $Y_{i}$ and $q_{i}$ cannot, however, be both timelike. Indeed, due to the reverse Cauchy-Schwarz inequality the product of two timelike vectors cannot be equal to zero, see [16]. Consequently, a non-lightlike solution of (0.32) must be spacelike. On the other hand, the dispersion relation can have a solution with $q^{2}=0$. For instance, it appears when the tensor $S_{i j}$ in (0.33) is antisymmetric. If the second solution of the dispersion relation is real the model describes birefringence. Rewrite (0.30) as

$$
q^{2}\left(Y^{2}-q^{2}\right)=(Y q)^{2}
$$

If this dispersion relation has a lightlike solution $q^{2}=0$, then (0.32) holds and we remain with

$$
q^{2}=Y^{2}
$$

Recall that we are looking for the solutions that satisfy the gauge condition $(Y q)=0$. Once more, the covectors $Y_{i}$ and $q_{i}$ cannot be timelike and orthonormal at the same time. Consequently they must be spacelike. So we have proved the following

Theorem: For an arbitrary skewon modification of a pseudo-Riemannian space, the non-null solution of the dispersion relation is spacelike.

Since the wave covector $q_{i}=(w, \mathbf{k})$ must describe a true (measurable) wave, such a wave would propagate with a velocity higher than the universal speed of light constant. This is in direct conflict with the special relativity paradigm. In the curved vacuum of GR, the skewon part of the constitutive tensor must consequently be completely ruled out.

Two short remarks should be added to this result:

1. Isotropic material. The considerations above remain completely unaltered when we deal with an isotropic medium with two standard parameters $\varepsilon$ and $\mu$. In this case, however, the wave velocity for the skewon modified medium only exceeds the value $\sqrt{\varepsilon \mu}$ instead of the universal speed of light. Such behavior is certainly not excluded by any fundamental principle. Thus the skewon part could exist in a material as a phenomenological quantity. It could for instance be useful for the description of dissipative media. Moreover, waves with velocity higher than $\sqrt{\varepsilon \mu}$ could point towards the presence of the skewon.

2. Euclidean signature. The considerations above also remain valid for a Riemannian manifold with the Euclidean signature. Here the only change will be the leading sine of the left hand side of Eq. (0.30). In this case, however, the ordinary Cauchy-Schwarz inequality immediately yields $q^{2}=0$. Thus the unique solution is $q_{i}=0$. Consequently any skewon modification of Euclidean space does not allow wave propagation.

\section{Acknowledgements}

I acknowledge the GIF grant No. 1078-107.14/2009 for financial support.

41, 1231 (1990)

[3] V. A. Kostelecky and M. Mewes, Phys. Rev. D 66, 056005
[1] F. Wilczek, Phys. Rev. Lett. 58, 1799 (1987).

[2] S. M. Carroll, G. B. Field and R. Jackiw, Phys. Rev. D 
(2002).

[4] C. Lammerzahl, A. Macias and H. Mueller, Phys. Rev. D 71, 025007 (2005).

[5] Y. Itin, Phys. Rev. D 70, 025012 (2004).

[6] Y. Itin, Gen. Rel. Grav. 40, 1219 (2008).

[7] F. W. Hehl and Yu. N. Obukhov, Foundations of Classical Electrodynamics: Charge, Flux, and Metric (Birkhäuser: Boston, MA, 2003).

[8] J.F. Nieves and P.B. Pal, Phys. Rev. D 39, 652 (1989).

[9] Y. N. Obukhov and F. W. Hehl, Phys. Rev. D 70, 125015 (2004).

[10] F. W. Hehl, Y. .N. Obukhov, G. F. Rubilar and M. Blago- jevic, Phys. Lett. A 347, 14 (2005).

[11] I. V. Lindell, A. Sihvola, "Skewon-Axion Medium and Soft-and-Hard/DB Boundary Condition", arXiv 1201.4738 (2012).

[12] V. Perlick, J. Math. Phys. 52, 042903 (2011).

[13] Y. Itin, Annals Phys. 327, 359 (2012). [gr-qc/0401016].

[14] Y. N. Obukhov, T. Fukui and G. F. Rubilar, Phys. Rev. D 62, 044050 (2000).

[15] Y. Itin, J. Phys. A 42, 475402 (2009).

[16] D. Giulini, Lect. Notes Phys. 702, 45 (2006). 